\title{
A strengthened Mulholland-type inequality with parameters
}

Aizhen Wang*, Qiliang Huang and Bicheng Yang

\section{"Correspondence:}

sxxmaths876@sina.com

Department of Mathematics,

Guangdong University of

Education, Guangzhou, Guangdong

510303, P.R. China

\begin{abstract}
By means of the way of weight coefficients, technique of real analysis, and Hermite-Hadamard's inequality, a strengthened version of the Mulholland-type inequality with the best possible constant factor and multi-parameters is given. The equivalent forms, the reverses, the operator expressions and a few particular cases are considered.
\end{abstract}

MSC: $26 \mathrm{D} 15 ; 47 \mathrm{~A} 07$

Keywords: Mulholland-type inequality; weight coefficient; equivalent form; reverse; operator

\section{Introduction}

If $p>1, \frac{1}{p}+\frac{1}{q}=1, a_{m}, b_{n} \geq 0, a=\left\{a_{m}\right\}_{m=1}^{\infty} \in l^{p}, b=\left\{b_{n}\right\}_{n=1}^{\infty} \in l^{q},\|a\|_{p}=\left(\sum_{m=1}^{\infty} a_{m}^{p}\right)^{\frac{1}{p}}>0$, $\|b\|_{q}>0$, then we have the following well-known Hardy-Hilbert's inequality with the best possible constant factor $\frac{\pi}{\sin (\pi / p)}(c f .[1]$, Theorem 315):

$$
\sum_{m=1}^{\infty} \sum_{n=1}^{\infty} \frac{a_{m} b_{n}}{m+n}<\frac{\pi}{\sin (\pi / p)}\|a\|_{p}\|b\|_{q}
$$

Also we have the following Mulholland's inequality similar to (1) with the same best possible constant factor $\frac{\pi}{\sin (\pi / p)}\left(c f\right.$. [2] or [1], Theorem 343, replacing $\frac{a_{m}}{n}, \frac{b_{n}}{n}$ by $\left.a_{m}, b_{n}\right)$ :

$$
\sum_{m=2}^{\infty} \sum_{n=2}^{\infty} \frac{a_{m} b_{n}}{\ln m n}<\frac{\pi}{\sin (\pi / p)}\left(\sum_{m=2}^{\infty} \frac{a_{m}^{p}}{m^{1-p}}\right)^{\frac{1}{p}}\left(\sum_{n=2}^{\infty} \frac{b_{n}^{q}}{n^{1-q}}\right)^{\frac{1}{q}} .
$$

Inequalities (1) and (2) are important in analysis and its applications (cf. [1, 3-8]).

In 1998, Gao and Yang [9] gave a strengthened version of (1) as follows:

$$
\begin{aligned}
\sum_{m=1}^{\infty} \sum_{n=1}^{\infty} \frac{a_{m} b_{n}}{m+n}< & \left\{\sum_{m=1}^{\infty}\left[\frac{\pi}{\sin (\pi / p)}-\frac{1-\gamma}{m^{1 / p}}\right] a_{m}^{p}\right\}^{\frac{1}{p}} \\
& \times\left\{\sum_{n=1}^{\infty}\left[\frac{\pi}{\sin (\pi / p)}-\frac{1-\gamma}{n^{1 / q}}\right] b_{n}^{q}\right\}^{\frac{1}{q}},
\end{aligned}
$$

where $1-\gamma=0.42278433^{+}$( $\gamma$ is Euler constant).

(c) 2015 Wang et al. This article is distributed under the terms of the Creative Commons Attribution 4.0 International License (http://creativecommons.org/licenses/by/4.0/), which permits unrestricted use, distribution, and reproduction in any medium, provided you give appropriate credit to the original author(s) and the source, provide a link to the Creative Commons license, and indicate if changes were made. 
Suppose that $\mu_{i}, v_{j}>0(i, j \in \mathbf{N}=\{1,2, \ldots\})$,

$$
U_{m}:=\sum_{i=1}^{m} \mu_{i}, \quad V_{n}:=\sum_{j=1}^{n} v_{j} \quad(m, n \in \mathbf{N})
$$

we have the following Hardy-Hilbert-type inequality (cf. [1], Theorem 321):

$$
\sum_{m=1}^{\infty} \sum_{n=1}^{\infty} \frac{\mu_{m}^{1 / q} v_{n}^{1 / p} a_{m} b_{n}}{U_{m}+V_{n}}<\frac{\pi}{\sin (\pi / p)}\|a\|_{p}\|b\|_{q} .
$$

For $\mu_{i}=v_{j}=1(i, j \in \mathbf{N})$, inequality (5) reduces to (1). Replacing $\mu_{m}^{1 / q} a_{m}$ and $v_{n}^{1 / p} b_{n}$ by $a_{m}$ and $b_{n}$ in (5), respectively, we obtain the equivalent form of (5) as follows:

$$
\sum_{m=1}^{\infty} \sum_{n=1}^{\infty} \frac{a_{m} b_{n}}{U_{m}+V_{n}}<\frac{\pi}{\sin \left(\frac{\pi}{p}\right)}\left(\sum_{m=1}^{\infty} \frac{a_{m}^{p}}{\mu_{m}^{p-1}}\right)^{\frac{1}{p}}\left(\sum_{n=1}^{\infty} \frac{b_{n}^{q}}{v_{n}^{q-1}}\right)^{\frac{1}{q}} .
$$

In 2015, Yang [10] gave an extension of (6) as follows: For $0<\lambda_{1}, \lambda_{2} \leq 1, \lambda_{1}+\lambda_{2}=\lambda$, we have

$$
\begin{aligned}
\sum_{m=1}^{\infty} & \sum_{n=1}^{\infty} \frac{a_{m} b_{n}}{\left(U_{m}+V_{n}\right)^{\lambda}} \\
& <B\left(\lambda_{1}, \lambda_{2}\right)\left[\sum_{m=1}^{\infty} \frac{U_{m}^{p\left(1-\lambda_{1}\right)-1} a_{m}^{p}}{\mu_{m}^{p-1}}\right]^{\frac{1}{p}}\left[\sum_{n=1}^{\infty} \frac{V_{n}^{q\left(1-\lambda_{2}-1\right)} b_{n}^{q}}{v_{n}^{q-1}}\right]^{\frac{1}{q}},
\end{aligned}
$$

where $B(u, v)$ is the beta function indicated by $(c f .[11])$

$$
B(u, v):=\int_{0}^{\infty} \frac{t^{u-1}}{(1+t)^{u+v}} d t \quad(u, v>0)
$$

In this paper, by using the way of weight coefficients, the technique of real analysis, and Hermite-Hadamard's inequality, a Mulholland-type inequality with the best possible constant factor $\frac{\pi}{\sin (\pi / p)}$ is given as follows: For $\mu_{1}=v_{1}=1,\left\{\mu_{m}\right\}_{m=1}^{\infty}$ and $\left\{v_{n}\right\}_{n=1}^{\infty}$ are decreasing, and $U_{\infty}=V_{\infty}=\infty$, we have

$$
\begin{aligned}
& \sum_{m=2}^{\infty} \sum_{n=2}^{\infty} \frac{a_{m} b_{n}}{\ln U_{m} V_{n}} \\
& \quad<\frac{\pi}{\sin (\pi / p)}\left[\sum_{m=2}^{\infty}\left(\frac{U_{m}}{\mu_{m+1}}\right)^{p-1} a_{m}^{p}\right]^{\frac{1}{p}}\left[\sum_{n=2}^{\infty}\left(\frac{V_{n}}{v_{n+1}}\right)^{q-1} b_{n}^{q}\right]^{\frac{1}{q}},
\end{aligned}
$$

which is an extension of (2) (Note: the series on the right-hand side of (9) are positive). Moreover, a strengthened version of (9) and some extended Mulholland-type inequalities with multi-parameters are obtained. The equivalent forms, the reverses, the operator expressions and a few particular cases are considered. 


\section{Some lemmas}

In the following, we make appointment that $p \neq 0,1, \frac{1}{p}+\frac{1}{q}=1,0<\lambda_{1}, \lambda_{2} \leq 1, \lambda_{1}+\lambda_{2}=\lambda$, $\mu_{i}, v_{j}>0(i, j \in \mathbf{N})$, with $\mu_{1}=v_{1}=1, U_{m}$ and $V_{n}$ are defined by (4), $a_{m}, b_{n} \geq 0,\|a\|_{p, \Phi_{\lambda}}:=$ $\left(\sum_{m=2}^{\infty} \Phi_{\lambda}(m) a_{m}^{p}\right)^{\frac{1}{p}}$ and $\|b\|_{q, \Psi_{\lambda}}:=\left(\sum_{n=2}^{\infty} \Psi_{\lambda}(n) b_{n}^{q}\right)^{\frac{1}{q}}$, where

$$
\begin{aligned}
& \Phi_{\lambda}(m):=\frac{\left(\ln U_{m}\right)^{p\left(1-\lambda_{1}\right)-1}}{U_{m}^{1-p} \mu_{m+1}^{p-1}}, \\
& \Psi_{\lambda}(n):=\frac{\left(\ln V_{n}\right)^{q\left(1-\lambda_{2}\right)-1}}{V_{n}^{1-q} v_{n+1}^{q-1}} \quad(m, n \in \mathbf{N} \backslash\{1\}) .
\end{aligned}
$$

Lemma 1 If $a \in \mathbf{R}, f(x)$ is continuous in $\left[a-\frac{1}{2}, a+\frac{1}{2}\right], f^{\prime}(x)$ is strictly increasing in $\left(a-\frac{1}{2}, a\right)$ and $\left(a, a+\frac{1}{2}\right)$, respectively, and

$$
\lim _{x \rightarrow a-} f^{\prime}(x)=f^{\prime}(a-0) \leq f^{\prime}(a+0)=\lim _{x \rightarrow a+} f^{\prime}(x),
$$

then we have the following Hermite-Hadamard's inequality (cf. [12]):

$$
f(a)<\int_{a-\frac{1}{2}}^{a+\frac{1}{2}} f(x) d x
$$

Proof Since $f^{\prime}(a-0)\left(\leq f^{\prime}(a+0)\right)$ is finite, we set a function $g(x)$ as follows:

$$
g(x):=f^{\prime}(a-0)(x-a)+f(a), \quad x \in\left[a-\frac{1}{2}, a+\frac{1}{2}\right] .
$$

In view of $f^{\prime}(x)$ being strictly increasing in $\left(a-\frac{1}{2}, a\right)$, then for $x \in\left(a-\frac{1}{2}, a\right),(f(x)-g(x))^{\prime}=$ $f^{\prime}(x)-f^{\prime}(a-0)<0$. Since $f(a)-g(a)=0$, it follows that $f(x)-g(x)>0, x \in\left(a-\frac{1}{2}, a\right)$. In the same way, we can obtain $f(x)-g(x)>0, x \in\left(a, a+\frac{1}{2}\right)$. Hence, we find

$$
\int_{a-\frac{1}{2}}^{a+\frac{1}{2}} f(x) d x>\int_{a-\frac{1}{2}}^{a+\frac{1}{2}} g(x) d x=f(a),
$$

namely (11) follows.

Example 1 If $\left\{\mu_{m}\right\}_{m=1}^{\infty}$ and $\left\{v_{n}\right\}_{n=1}^{\infty}$ are also decreasing, we set $\mu(t):=\mu_{m}, t \in(m-1, m]$ $(m \in \mathbf{N}) ; v(t):=v_{n}, t \in(n-1, n](n \in \mathbf{N})$,

$$
U(x):=\int_{0}^{x} \mu(t) d t \quad(x \geq 0), \quad V(y):=\int_{0}^{y} v(t) d t \quad(y \geq 0) .
$$

Then it follows that $U(m)=U_{m}, V(n)=V_{n}(m, n \in \mathbf{N}), U(\infty)=U_{\infty}, V(\infty)=V_{\infty}$ and

$$
\begin{aligned}
& U^{\prime}(x)=\mu(x)=\mu_{m} \quad(x \in(m-1, m)), \\
& V^{\prime}(y)=v(y)=v_{n} \quad(y \in(n-1, n)) .
\end{aligned}
$$

For fixed $m, n \in \mathbf{N} \backslash\{1\}$, we also set a function $f(x)$ as follows:

$$
f(x)=\frac{\ln ^{\lambda_{2}-1} V(x)}{V(x)\left(\ln U_{m}+\ln V(x)\right)^{\lambda}}, \quad x \in\left[n-\frac{1}{2}, n+\frac{1}{2}\right] .
$$


Then $f(x)$ in continuous in $\left[n-\frac{1}{2}, n+\frac{1}{2}\right]$. For $x \in\left(n-\frac{1}{2}, n\right)(n \in \mathbf{N} \backslash\{1\})$, we find

$$
\begin{aligned}
f^{\prime}(x)= & -\left[\frac{\ln ^{\lambda_{2}-1} V(x)}{V(x)}+\frac{\lambda \ln ^{\lambda_{2}-1} V(x)}{\ln U_{m}+\ln V(x)}+\frac{1-\lambda_{2}}{V^{2-\lambda_{2}}(x)}\right] \\
& \times \frac{v_{n}}{V(x)\left(\ln U_{m}+\ln V(x)\right)^{\lambda}} .
\end{aligned}
$$

Since $1-\lambda_{2} \geq 0$, it follows that $f^{\prime}(x)(<0)$ is strictly increasing in $\left(n-\frac{1}{2}, n\right)$ and

$$
\begin{aligned}
\lim _{x \rightarrow n_{-}} f^{\prime}(x)= & f^{\prime}(n-0) \\
= & -\left[\frac{\ln ^{\lambda_{2}-1} V_{n}}{V_{n}}+\frac{\lambda \ln ^{\lambda_{2}-1} V_{n}}{\ln U_{m}+\ln V_{n}}+\frac{1-\lambda_{2}}{V_{n}^{2-\lambda_{2}}}\right] \\
& \times \frac{v_{n}}{V_{n}\left(\ln U_{m}+\ln V_{n}\right)^{\lambda}} .
\end{aligned}
$$

In the same way, for $x \in\left(n, n+\frac{1}{2}\right)$, we find

$$
\begin{aligned}
f^{\prime}(x)= & -\left[\frac{\ln ^{\lambda_{2}-1} V(x)}{V(x)}+\frac{\lambda \ln ^{\lambda_{2}-1} V(x)}{\ln U_{m}+\ln V(x)}+\frac{1-\lambda_{2}}{V^{2-\lambda_{2}}(x)}\right] \\
& \times \frac{v_{n+1}}{V(x)\left(\ln U_{m}+\ln V(x)\right)^{\lambda}}
\end{aligned}
$$

$f^{\prime}(x)(<0)$ is strictly increasing in $\left(n, n+\frac{1}{2}\right)$. In view of $v_{n+1} \leq v_{n}$, it follows that $\lim _{x \rightarrow n+} f^{\prime}(x)=f^{\prime}(n+0) \geq f^{\prime}(n-0)$. Then by (11) we have

$$
f(n)<\int_{n-\frac{1}{2}}^{n+\frac{1}{2}} f(x) d x=\int_{n-\frac{1}{2}}^{n+\frac{1}{2}} \frac{\ln ^{\lambda_{2}-1} V(x)}{V(x)\left(\ln U_{m}+\ln V(x)\right)^{\lambda}} d x
$$

Definition 1 Define the following weight coefficients:

$$
\begin{aligned}
& \omega\left(\lambda_{2}, m\right):=\sum_{n=2}^{\infty} \frac{1}{\ln ^{\lambda}\left(U_{m} V_{n}\right)} \frac{v_{n+1} \ln ^{\lambda_{1}} U_{m}}{V_{n} \ln ^{1-\lambda_{2}} V_{n}}, \quad m \in \mathbf{N} \backslash\{1\}, \\
& \varpi\left(\lambda_{1}, n\right):=\sum_{m=2}^{\infty} \frac{1}{\ln ^{\lambda}\left(U_{m} V_{n}\right)} \frac{\mu_{m+1} \ln ^{\lambda_{2}} V_{n}}{U_{m} \ln ^{1-\lambda_{1}} U_{m}}, \quad n \in \mathbf{N} \backslash\{1\} .
\end{aligned}
$$

Lemma 2 If $\left\{\mu_{m}\right\}_{m=1}^{\infty}$ and $\left\{v_{n}\right\}_{n=1}^{\infty}$ are decreasing, and $U_{\infty}=V_{\infty}=\infty$, then we have the following inequalities:

$$
\begin{gathered}
\omega\left(\lambda_{2}, m\right)<B\left(\lambda_{1}, \lambda_{2}\right)\left(1-\frac{\theta_{1}}{\ln ^{\lambda_{2}} U_{m}}\right) \\
\left(0<\lambda_{2} \leq 1, \lambda_{1}>0 ; m \in \mathbf{N} \backslash\{1\}\right), \\
\varpi\left(\lambda_{1}, n\right)<B\left(\lambda_{1}, \lambda_{2}\right)\left(1-\frac{\theta_{2}}{\ln ^{\lambda_{1}} V_{n}}\right) \\
\left(0<\lambda_{1} \leq 1, \lambda_{2}>0 ; n \in \mathbf{N} \backslash\{1\}\right),
\end{gathered}
$$


where

$$
\begin{aligned}
& \theta_{1}:=\frac{1}{B\left(\lambda_{1}, \lambda_{2}\right)} \frac{\ln ^{\lambda_{2}}\left(1+v_{2} / 2\right)}{\lambda_{2}\left[1+\frac{\ln \left(1+\nu_{2} / 2\right)}{\ln \left(1+\mu_{2} / 2\right)} \lambda^{\lambda}\right.}, \\
& \theta_{2}:=\frac{1}{B\left(\lambda_{1}, \lambda_{2}\right)} \frac{\ln ^{\lambda_{1}}\left(1+\mu_{2} / 2\right)}{\lambda_{1}\left[1+\frac{\ln \left(1+\mu_{2} / 2\right)}{\ln \left(1+\nu_{2} / 2\right)}\right]^{\lambda} .}
\end{aligned}
$$

Proof Since for $x \in\left(n-\frac{1}{2}, n+\frac{1}{2}\right) \backslash\{n\}, v_{n+1} \leq V^{\prime}(x)$, by (13) we find

$$
\begin{aligned}
\omega\left(\lambda_{2}, m\right)< & \sum_{n=2}^{\infty} v_{n+1} \int_{n-\frac{1}{2}}^{n+\frac{1}{2}} \frac{\ln ^{\lambda_{1}} U_{m} \ln ^{\lambda_{2}-1} V(x)}{V(x)\left(\ln U_{m}+\ln V(x)\right)^{\lambda}} d x \\
\leq & \sum_{n=2}^{\infty} \int_{n-\frac{1}{2}}^{n+\frac{1}{2}} \frac{\ln ^{\lambda_{1}} U_{m} \ln ^{\lambda_{2}-1} V(x)}{V(x)\left(\ln U_{m}+\ln V(x)\right)^{\lambda}} V^{\prime}(x) d x \\
= & \int_{\frac{3}{2}}^{\infty} \frac{\ln ^{\lambda_{1}} U_{m} \ln ^{\lambda_{2}-1} V(x)}{V(x)\left(\ln U_{m}+\ln V(x)\right)^{\lambda}} V^{\prime}(x) d x \\
= & \int_{1}^{\infty} \frac{\ln ^{\lambda_{1}} U_{m} \ln ^{\lambda_{2}-1} V(x)}{V(x)\left(\ln U_{m}+\ln V(x)\right)^{\lambda}} V^{\prime}(x) d x \\
& -\int_{1}^{\frac{3}{2}} \frac{\ln { }^{\lambda_{1}} U_{m} \ln ^{\lambda_{2}-1} V(x)}{V(x)\left(\ln U_{m}+\ln V(x)\right)^{\lambda}} V^{\prime}(x) d x .
\end{aligned}
$$

Setting $t=\frac{\ln V(x)}{\ln U_{m}}$, we obtain $\frac{V^{\prime}(x)}{V(x)} d x=\ln U_{m} d t$ and

$$
\begin{aligned}
\omega\left(\lambda_{2}, m\right) & <\int_{0}^{\infty} \frac{1}{(1+t)^{\lambda}} t^{\lambda_{2}-1} d t-\int_{1}^{\frac{3}{2}} \frac{\ln ^{\lambda_{1}} U_{m}}{\left(\ln U_{m}+\ln V(x)\right)^{\lambda}} \frac{\ln ^{\lambda_{2}-1} V(x)}{V(x)} V^{\prime}(x) d x \\
& =B\left(\lambda_{1}, \lambda_{2}\right)(1-\theta(m)),
\end{aligned}
$$

where

$$
\theta(m):=\frac{1}{B\left(\lambda_{1}, \lambda_{2}\right)} \int_{1}^{\frac{3}{2}} \frac{\ln ^{\lambda_{1}} U_{m}}{\left(\ln U_{m}+\ln V(x)\right)^{\lambda}} \frac{\ln ^{\lambda_{2}-1} V(x)}{V(x)} V^{\prime}(x) d x .
$$

We find

$$
\begin{aligned}
\theta(m) & \geq \frac{1}{B\left(\lambda_{1}, \lambda_{2}\right)} \frac{\ln ^{\lambda_{1}} U_{m}}{\left(\ln U_{m}+\ln V\left(\frac{3}{2}\right)\right)^{\lambda}} \int_{1}^{\frac{3}{2}} \frac{\ln ^{\lambda_{2}-1} V(x)}{V(x)} V^{\prime}(x) d x \\
& =\frac{1}{B\left(\lambda_{1}, \lambda_{2}\right)} \frac{\ln ^{\lambda_{1}} U_{m}}{\lambda_{2}\left(\ln U_{m}+\ln V\left(\frac{3}{2}\right)\right)^{\lambda}} \ln ^{\lambda_{2}} V\left(\frac{3}{2}\right) \\
& =\frac{1}{B\left(\lambda_{1}, \lambda_{2}\right)} \frac{\ln ^{\lambda_{2}}\left(1+v_{2} / 2\right)}{\lambda_{2}\left(1+\frac{\ln \left(1+v_{2} / 2\right)}{\ln U_{m}}\right)^{\lambda}} \frac{1}{\ln ^{\lambda_{2}} U_{m}} \\
& \geq \frac{1}{B\left(\lambda_{1}, \lambda_{2}\right)} \frac{\ln ^{\lambda_{2}}\left(1+v_{2} / 2\right)}{\lambda_{2}\left(1+\frac{\ln \left(1+v_{2} / 2\right)}{\ln U_{2}}\right)^{\lambda}} \frac{1}{\ln ^{\lambda_{2}} U_{m}}=\frac{\theta_{1}}{\ln ^{\lambda_{2}} U_{m}} .
\end{aligned}
$$

Hence, by (20), we have (16) and (18). In the same way, we obtain (17) and (19).

Note For example, $\mu_{n}, v_{n}=\frac{1}{n^{\sigma}}(0 \leq \sigma \leq 1)$ are satisfied the assumptions of Lemma 2. 
Lemma 3 With the assumptions of Lemma 2, (i) for $m, n \in \mathbf{N} \backslash\{1\}$, we have

$$
\begin{array}{ll}
B\left(\lambda_{1}, \lambda_{2}\right)\left(1-\theta\left(\lambda_{2}, m\right)\right)<\omega\left(\lambda_{2}, m\right) & \left(0<\lambda_{2} \leq 1, \lambda_{1}>0\right), \\
B\left(\lambda_{1}, \lambda_{2}\right)\left(1-\vartheta\left(\lambda_{1}, n\right)\right)<\varpi\left(\lambda_{1}, n\right) & \left(0<\lambda_{1} \leq 1, \lambda_{2}>0\right),
\end{array}
$$

where

$$
\begin{aligned}
\theta\left(\lambda_{2}, m\right) & =\frac{1}{B\left(\lambda_{1}, \lambda_{2}\right)} \frac{\ln ^{\lambda_{2}}\left(1+v_{2}\right)}{\lambda_{2}\left[1+\frac{\ln \left(1+\theta(m) v_{2}\right)}{\ln U_{m}}\right]^{\lambda}} \frac{1}{\ln ^{\lambda_{2}} U_{m}} \\
& =O\left(\frac{1}{\ln ^{\lambda_{2}} U_{m}}\right) \in(0,1) \quad(\theta(m) \in(0,1)), \\
\vartheta\left(\lambda_{1}, n\right) & =\frac{1}{B\left(\lambda_{1}, \lambda_{2}\right)} \frac{\ln ^{\lambda_{1}}\left(1+\mu_{2}\right)}{\lambda_{1}\left[1+\frac{\ln \left(1+\vartheta(n) \mu_{2}\right)}{\ln V_{n}}\right]^{\lambda}} \frac{1}{\ln ^{\lambda_{1}} V_{n}} \\
& =O\left(\frac{1}{\ln ^{\lambda_{1}} V_{n}}\right) \in(0,1) \quad(\vartheta(n) \in(0,1)) ;
\end{aligned}
$$

(ii) for any $a>0$, we have

$$
\begin{aligned}
& \sum_{m=2}^{\infty} \frac{\mu_{m+1}}{U_{m} \ln ^{1+a} U_{m}}=\frac{1}{a}\left[\frac{1}{\ln ^{a}\left(1+\mu_{2}\right)}+a O(1)\right], \\
& \sum_{n=2}^{\infty} \frac{v_{n+1}}{V_{n} \ln ^{1+a} V_{n}}=\frac{1}{a}\left[\frac{1}{\ln ^{a}\left(1+v_{2}\right)}+a \widetilde{O}(1)\right] .
\end{aligned}
$$

Proof Since by Example 1, $f(x)$ is strictly decreasing in $[n, n+1]$, then we find

$$
\begin{aligned}
\omega\left(\lambda_{2}, m\right)> & \sum_{n=2}^{\infty} \int_{n}^{n+1} v_{n+1} \frac{\ln ^{\lambda_{1}} U_{m} \ln ^{\lambda_{2}-1} V(x)}{V(x)\left(\ln U_{m}+\ln V(x)\right)^{\lambda}} d x \\
= & \int_{2}^{\infty} \frac{\ln ^{\lambda_{1}} U_{m} \ln ^{\lambda_{2}-1} V(x)}{V(x)\left(\ln U_{m}+\ln V(x)\right)^{\lambda}} V^{\prime}(x) d x \\
= & \int_{1}^{\infty} \frac{\ln ^{\lambda_{1}} U_{m} \ln ^{\lambda_{2}-1} V(x)}{V(x)\left(\ln U_{m}+\ln V(x)\right)^{\lambda}} V^{\prime}(x) d x \\
& -\int_{1}^{2} \frac{\ln ^{\lambda_{1}} U_{m} \ln ^{\lambda_{2}-1} V(x)}{V(x)\left(\ln U_{m}+\ln V(x)\right)^{\lambda}} V^{\prime}(x) d x \\
= & B\left(\lambda_{1}, \lambda_{2}\right)\left(1-\theta\left(\lambda_{2}, m\right)\right),
\end{aligned}
$$

where

$$
\theta\left(\lambda_{2}, m\right):=\frac{1}{B\left(\lambda_{1}, \lambda_{2}\right)} \int_{1}^{2} \frac{V^{\prime}(x) \ln ^{\lambda_{1}} U_{m} \ln ^{\lambda_{2}-1} V(x)}{V(x)\left(\ln U_{m}+\ln V(x)\right)^{\lambda}} d x \in(0,1) .
$$

There exists $\theta(m) \in(0,1)$ such that

$$
\begin{aligned}
\theta\left(\lambda_{2}, m\right)= & \frac{1}{B\left(\lambda_{1}, \lambda_{2}\right)} \frac{\ln ^{\lambda_{1}} U_{m}}{\left[\ln U_{m}+\ln V(1+\theta(m))\right]^{\lambda}} \\
& \times \int_{1}^{2} \frac{\ln ^{\lambda_{2}-1} V(x)}{V(x)} V^{\prime}(x) d x
\end{aligned}
$$




$$
\begin{aligned}
& =\frac{1}{B\left(\lambda_{1}, \lambda_{2}\right)} \frac{\ln ^{\lambda_{1}} U_{m} \ln ^{\lambda_{2}}\left(1+v_{2}\right)}{\lambda_{2}\left[\ln U_{m}+\ln V(1+\theta(m))\right]^{\lambda}} \\
& =\frac{1}{B\left(\lambda_{1}, \lambda_{2}\right)} \frac{\ln ^{\lambda_{2}}\left(1+v_{2}\right)}{\lambda_{2}\left[1+\frac{\ln \left(1+\theta(m) v_{2}\right)}{\ln U_{m}}\right]^{\lambda}} \frac{1}{\ln ^{\lambda_{2}} U_{m}} .
\end{aligned}
$$

Since we obtain

$$
0<\theta\left(\lambda_{2}, m\right) \leq \frac{\ln ^{\lambda_{2}}\left(1+v_{2}\right)}{\lambda_{2} B\left(\lambda_{1}, \lambda_{2}\right)} \frac{1}{\ln ^{\lambda_{2}} U_{m}},
$$

namely $\theta\left(\lambda_{2}, m\right)=O\left(\frac{1}{\ln ^{2} 2 U_{m}}\right)$, we have (22). In the same way, we obtain (23).

For $a>0$, we find

$$
\begin{aligned}
& \sum_{m=2}^{\infty} \frac{\mu_{m+1}}{U_{m} \ln ^{1+a} U_{m}} \leq \sum_{m=2}^{\infty} \frac{\mu_{m}}{U_{m} \ln ^{1+a} U_{m}} \\
&=\frac{\mu_{2}}{U_{2} \ln ^{1+a} U_{2}}+\sum_{m=3}^{\infty} \frac{\mu_{m}}{U_{m} \ln ^{1+a} U_{m}} \\
&=\frac{\mu_{2}}{U_{2} \ln ^{1+a} U_{2}}+\sum_{m=3}^{\infty} \int_{m-1}^{m} \frac{U^{\prime}(x)}{U_{m} \ln ^{1+a} U_{m}} d x \\
&<\frac{\mu_{2}}{U_{2} \ln ^{1+a} U_{2}}+\sum_{m=3}^{\infty} \int_{m-1}^{m} \frac{U^{\prime}(x)}{U(x) \ln ^{1+a} U(x)} d x \\
&=\frac{\mu_{2}}{U_{2} \ln ^{1+a} U_{2}}+\int_{2}^{\infty} \frac{U^{\prime}(x)}{U(x) \ln ^{1+a} U(x)} d x \\
&=\frac{\mu_{2}}{\left(1+\mu_{2}\right) \ln ^{1+a}\left(1+\mu_{2}\right)}+\frac{1}{a \ln ^{a}\left(1+\mu_{2}\right)} \\
&=\frac{1}{a}\left(\frac{1}{\ln ^{a}\left(1+\mu_{2}\right)}+\frac{a \mu_{2}}{\left(1+\mu_{2}\right) \ln ^{1+a}\left(1+\mu_{2}\right)}\right) \\
&=\sum_{m=2}^{\infty} \int_{m}^{m+1} \frac{U^{\prime}(x) d x}{U_{m} \ln ^{1+a} U_{m}} \\
&>\sum_{m=2}^{\infty} \int_{m}^{m+1} \frac{U^{\prime}(x)}{U(x) \ln ^{1+a} U(x)} d x \\
& \sum_{m=2}^{\infty} \frac{U^{\prime}(x) d x}{\mu_{m} \ln ^{1+a} U_{m}}=\frac{1}{a \ln ^{a}\left(1+\mu_{2}\right)}
\end{aligned}
$$

Hence we have (26). In the same way, we have (27).

\section{Main results and operator expressions}

We also set

$$
\begin{aligned}
& \widetilde{\Phi}_{\lambda}(m):=\omega\left(\lambda_{2}, m\right) \frac{\left(\ln U_{m}\right)^{p\left(1-\lambda_{1}\right)-1}}{U_{m}^{1-p} \mu_{m+1}^{p-1}}, \\
& \widetilde{\Psi}_{\lambda}(n):=\varpi\left(\lambda_{1}, n\right) \frac{\left(\ln V_{n}\right)^{q\left(1-\lambda_{2}\right)-1}}{V_{n}^{1-q} v_{n+1}^{q-1}} \quad(m, n \in \mathbf{N} \backslash\{1\}) .
\end{aligned}
$$


Theorem 1 (i) For $p>1$, we have the following equivalent inequalities:

$$
\begin{aligned}
& I:=\sum_{n=2}^{\infty} \sum_{m=2}^{\infty} \frac{a_{m} b_{n}}{\ln ^{\lambda}\left(U_{m} V_{n}\right)} \leq\|a\|_{p, \widetilde{\Phi}_{\lambda}}\|b\|_{q, \widetilde{\Psi}_{\lambda},} \\
& J:=\left\{\sum_{n=2}^{\infty} \frac{v_{n+1} \ln ^{p \lambda_{2}-1} V_{n}}{\left(\varpi\left(\lambda_{1}, n\right)\right)^{p-1} V_{n}}\left[\sum_{m=2}^{\infty} \frac{a_{m}}{\ln ^{\lambda}\left(U_{m} V_{n}\right)}\right]^{p}\right\}^{\frac{1}{p}} \leq\|a\|_{p, \widetilde{\Phi}_{\lambda}} .
\end{aligned}
$$

(ii) For $0<p<1$ (or $p<0)$, we have the equivalent reverses of (29) and (30).

Proof (i) By Hölder's inequality with weight (cf. [12]) and (15), we have

$$
\begin{aligned}
{\left[\sum_{m=2}^{\infty} \frac{a_{m}}{\ln ^{\lambda}\left(U_{m} V_{n}\right)}\right]^{p}=} & {\left[\sum_{m=2}^{\infty} \frac{1}{\ln ^{\lambda}\left(U_{m} V_{n}\right)}\left(\frac{U_{m}^{1 / q}\left(\ln U_{m}\right)^{\left(1-\lambda_{1}\right) / q} v_{n+1}^{1 / p}}{\left(\ln V_{n}\right)^{\left(1-\lambda_{2}\right) / p} \mu_{m+1}^{1 / q}} a_{m}\right)\right.} \\
& \left.\times\left(\frac{\left(\ln V_{n}\right)^{\left(1-\lambda_{2}\right) / p} \mu_{m+1}^{1 / q}}{U_{m}^{1 / q}\left(\ln U_{m}\right)^{\left(1-\lambda_{1}\right) / q} v_{n+1}^{1 / p}}\right)\right]^{p} \\
\leq & \sum_{m=2}^{\infty} \frac{1}{\ln ^{\lambda}\left(U_{m} V_{n}\right)} \frac{U_{m}^{p-1}\left(\ln U_{m}\right)^{\left(1-\lambda_{1}\right) p / q} v_{n+1}}{\left(\ln V_{n}\right)^{1-\lambda_{2}} \mu_{m+1}^{p / q}} a_{m}^{p} \\
& \times\left[\sum_{m=2}^{\infty} \frac{1}{\ln \left(U_{m} V_{n}\right)} \frac{\left(\ln V_{n}\right)^{\left(1-\lambda_{2}\right)(q-1)} \mu_{m+1}}{U_{m}\left(\ln U_{m}\right)^{1-\lambda_{1}} v_{n+1}^{q-1}}\right]^{p-1} \\
= & \frac{\left(\varpi\left(\lambda_{1}, n\right)\right)^{p-1} V_{n}}{\left(\ln V_{n}\right)^{p \lambda_{2}-1} v_{n+1}} \sum_{m=2}^{\infty} \frac{v_{n+1} U_{m}^{p-1}\left(\ln U_{m}\right)^{\left(1-\lambda_{1}\right)(p-1)} a_{m}^{p}}{\ln ^{\lambda}\left(U_{m} V_{n}\right) V_{n}\left(\ln V_{n}\right)^{1-\lambda_{2}} \mu_{m+1}^{p-1}} .
\end{aligned}
$$

Then by (14) we find

$$
\begin{aligned}
J & \leq\left[\sum_{n=2}^{\infty} \sum_{m=2}^{\infty} \frac{v_{n+1}}{\ln ^{\lambda}\left(U_{m} V_{n}\right)} \frac{U_{m}^{p-1}\left(\ln U_{m}\right)^{\left(1-\lambda_{1}\right)(p-1)}}{V_{n}\left(\ln V_{n}\right)^{1-\lambda_{2}} \mu_{m+1}^{p-1}} a_{m}^{p}\right]^{\frac{1}{p}} \\
& =\left[\sum_{m=2}^{\infty} \sum_{n=2}^{\infty} \frac{v_{n+1}\left(\ln U_{m}\right)^{\lambda_{1}}}{\ln ^{\lambda}\left(U_{m} V_{n}\right)} \frac{U_{m}^{p-1}\left(\ln U_{m}\right)^{p\left(1-\lambda_{1}\right)-1}}{V_{n}\left(\ln V_{n}\right)^{1-\lambda_{2}} \mu_{m+1}^{p-1}} a_{m}^{p}\right]^{\frac{1}{p}} \\
& =\left[\sum_{m=2}^{\infty} \omega\left(\lambda_{2}, m\right) \frac{\left(\ln U_{m}\right)^{p\left(1-\lambda_{1}\right)-1}}{U_{m}^{1-p} \mu_{m+1}^{p-1}} a_{m}^{p}\right]^{\frac{1}{p}},
\end{aligned}
$$

and then (30) follows.

By Hölder's inequality ( $c f .[12])$, we have

$$
\begin{aligned}
I= & \sum_{n=2}^{\infty}\left[\frac{\left(\ln V_{n}\right)^{\lambda_{2}-\frac{1}{p}} v_{n+1}^{1 / p}}{\left(\varpi\left(\lambda_{1}, n\right)\right)^{\frac{1}{q}} V_{n}^{\frac{1}{p}}} \sum_{m=1}^{\infty} \frac{a_{m}}{\ln ^{\lambda}\left(U_{m} V_{n}\right)}\right] \\
& \times\left[\left(\varpi\left(\lambda_{1}, n\right)\right)^{\frac{1}{q}} \frac{\left(\ln V_{n}\right)^{\frac{1}{p}-\lambda_{2}}}{V_{n}^{\frac{-1}{p}} v_{n+1}^{\frac{1}{p}}} b_{n}\right] \leq J\|b\|_{q, \widetilde{\Psi}_{\lambda} .}
\end{aligned}
$$

Then by (30) we have (29). 
On the other hand, assuming that (29) is valid, we set

$$
b_{n}:=\frac{\left(\ln V_{n}\right)^{p \lambda_{2}-1} v_{n+1}}{\left(\varpi\left(\lambda_{1}, n\right)\right)^{p-1} V_{n}}\left[\sum_{m=2}^{\infty} \frac{a_{m}}{\ln ^{\lambda}\left(U_{m} V_{n}\right)}\right]^{p-1}, \quad n \in \mathbf{N} \backslash\{1\} .
$$

Then we find $J^{p}=\|b\|_{q, \widetilde{\Psi}_{\lambda}}^{q}$. If $J=0$, then (30) is trivially valid; if $J=\infty$, then, by (32), (30) takes the form of equality. Suppose that $0<J<\infty$. By (29), it follows that

$$
\begin{aligned}
& \|b\|_{q, \widetilde{\Psi}_{\lambda}}^{q}=J^{p}=I \leq\|a\|_{p, \widetilde{\Phi}_{\lambda}}\|b\|_{q, \widetilde{\Psi}_{\lambda}}, \\
& \|b\|_{q, \widetilde{\Psi}_{\lambda}}^{q-1}=J \leq\|a\|_{p, \widetilde{\Phi}_{\lambda}},
\end{aligned}
$$

and then (30) follows, which is equivalent to (29).

(ii) For $0<p<1$ (or $p<0$ ), by the reverse Hölder's inequality with weight ( $c f$. [12]) and (15), we obtain the reverse of (31) (or (31)), then we have the reverse of (32), and then the reverse of (30) follows. By Hölder's inequality ( $c f$. [12]), we have the reverse of (33) and then by the reverse of (30), the reverse of (29) follows.

On the other hand, assuming that the reverse of (29) is valid, we set $b_{n}$ as (34). Then we find $J^{p}=\|b\|_{q, \widetilde{\Psi}_{\lambda}}^{q}$. If $J=\infty$, then the reverse of (30) is trivially valid; if $J=0$, then, by the reverse of (32), (30) takes the form of equality $(=0)$. Suppose that $0<J<\infty$. By the reverse of (29), it follows that the reverses of (35) and (36) are valid, and then the reverse of (30) follows, which is equivalent to the reverse of (29).

Setting

$$
\begin{aligned}
& \Omega_{\lambda}(m):=\left(1-\frac{\theta_{1}}{\ln ^{\lambda_{2}} U_{m}}\right) \frac{\left(\ln U_{m}\right)^{p\left(1-\lambda_{1}\right)-1}}{U_{m}^{1-p} \mu_{m+1}^{p-1}}, \\
& \digamma_{\lambda}(n):=\left(1-\frac{\theta_{2}}{\ln ^{\lambda_{1}} V_{n}}\right) \frac{\left(\ln V_{n}\right)^{q\left(1-\lambda_{2}\right)-1}}{V_{n}^{1-q} v_{n+1}^{q-1}} \quad(m, n \in \mathbf{N} \backslash\{1\}),
\end{aligned}
$$

we have the following.

Theorem 2 If $p>1,\left\{\mu_{m}\right\}_{m=1}^{\infty}$ and $\left\{v_{n}\right\}_{n=1}^{\infty}$ are decreasing, $U_{\infty}=V_{\infty}=\infty,\|a\|_{p, \Phi_{\lambda}} \in \mathbf{R}_{+}$and $\|b\|_{q, \Psi_{\lambda}} \in \mathbf{R}_{+}$, then we have the following equivalent inequalities:

$$
\begin{aligned}
& \sum_{n=2}^{\infty} \sum_{m=2}^{\infty} \frac{a_{m} b_{n}}{\ln ^{\lambda}\left(U_{m} V_{n}\right)}<B\left(\lambda_{1}, \lambda_{2}\right)\|a\|_{p, \Omega_{\lambda}}\|b\|_{q, \digamma_{\lambda},} \\
& J_{1}:=\left\{\sum_{n=2}^{\infty} \frac{v_{n+1} \ln ^{p \lambda_{2}-1} V_{n}}{\left(1-\frac{\theta_{2}}{\ln ^{\lambda} V_{n}}\right)^{p-1} V_{n}}\left[\sum_{m=2}^{\infty} \frac{a_{m}}{\ln ^{\lambda}\left(U_{m} V_{n}\right)}\right]^{p}\right\}^{\frac{1}{p}}<B\left(\lambda_{1}, \lambda_{2}\right)\|a\|_{p, \Omega_{\lambda},}
\end{aligned}
$$

where the constant factor $B\left(\lambda_{1}, \lambda_{2}\right)$ is the best possible.

Proof Using (16) and (17) in (29) and (30), since

$$
\begin{aligned}
& \left(\omega\left(\lambda_{2}, m\right)\right)^{\frac{1}{p}}<\left(B\left(\lambda_{1}, \lambda_{2}\right)\right)^{\frac{1}{p}}\left(1-\frac{\theta_{1}}{\ln ^{\lambda_{2}} U_{m}}\right)^{\frac{1}{p}} \quad(p>1), \\
& \left(\varpi\left(\lambda_{1}, n\right)\right)^{\frac{1}{q}}<\left(B\left(\lambda_{1}, \lambda_{2}\right)\right)^{\frac{1}{q}}\left(1-\frac{\theta_{2}}{\ln ^{\lambda_{1}} V_{n}}\right)^{\frac{1}{q}} \quad(q>1)
\end{aligned}
$$


and

$$
\frac{1}{\left(B\left(\lambda_{1}, \lambda_{2}\right)\right)^{p-1}\left(1-\frac{\theta_{2}}{\ln ^{\lambda_{1} V_{n}}}\right)^{p-1}}<\frac{1}{\left(\varpi\left(\lambda_{1}, n\right)\right)^{p-1}} \quad(p>1)
$$

we obtain equivalent inequalities (38) and (39).

For $\varepsilon \in\left(0, p \lambda_{1}\right)$, we set $\widetilde{\lambda}_{1}=\lambda_{1}-\frac{\varepsilon}{p}(\in(0,1)), \tilde{\lambda}_{2}=\lambda_{2}+\frac{\varepsilon}{p}(>0)$, and

$$
\begin{aligned}
& \widetilde{a}_{m}:=\frac{\mu_{m+1}}{U_{m}} \ln ^{\tilde{\lambda}_{1}-1} U_{m}=\frac{\mu_{m+1}}{U_{m}} \ln ^{\lambda_{1}-\frac{\varepsilon}{p}-1} U_{m}, \\
& \tilde{b}_{n}=\frac{v_{n+1}}{V_{n}} \ln ^{\tilde{\lambda}_{2}-\varepsilon-1} V_{n}=\frac{v_{n+1}}{V_{n}} \ln ^{\lambda_{2}-\frac{\varepsilon}{q}-1} V_{n} .
\end{aligned}
$$

Then, by (26), (27) and (23), we have

$$
\begin{aligned}
& \|\widetilde{a}\|_{p, \Omega_{\lambda}}\|\widetilde{b}\|_{q, \digamma_{\lambda}} \\
& \leq\|\tilde{a}\|_{p, \Phi_{\lambda}}\|\tilde{b}\|_{q, \Psi_{\lambda}} \\
& =\left(\sum_{m=2}^{\infty} \frac{\mu_{m+1}}{U_{m} \ln ^{1+\varepsilon} U_{m}}\right)^{\frac{1}{p}}\left(\sum_{n=2}^{\infty} \frac{v_{n+1}}{V_{n} \ln ^{1+\varepsilon} V_{n}}\right)^{\frac{1}{q}} \\
& =\frac{1}{\varepsilon}\left[\frac{1}{\ln ^{\varepsilon}\left(1+\mu_{2}\right)}+\varepsilon O(1)\right]^{\frac{1}{p}}\left[\frac{1}{\ln ^{\varepsilon}\left(1+v_{2}\right)}+\varepsilon \widetilde{O}(1)\right]^{\frac{1}{q}} \text {, } \\
& \widetilde{I}:=\sum_{n=2}^{\infty} \sum_{m=2}^{\infty} \frac{\widetilde{a}_{m} \widetilde{b}_{n}}{\ln ^{\lambda}\left(U_{m} V_{n}\right)} \\
& =\sum_{n=2}^{\infty}\left[\sum_{m=2}^{\infty} \frac{1}{\ln ^{\lambda}\left(U_{m} V_{n}\right)} \frac{\mu_{m+1} \ln ^{\tilde{\lambda}_{2}} V_{n}}{U_{m} \ln ^{1-\tilde{\lambda}_{1}} U_{m}}\right] \frac{v_{n+1}}{V_{n} \ln ^{\varepsilon+1} V_{n}} \\
& =\sum_{n=2}^{\infty} \varpi\left(\tilde{\lambda}_{1}, n\right) \frac{v_{n+1}}{V_{n} \ln ^{\varepsilon+1} V_{n}} \\
& \geq B\left(\widetilde{\lambda}_{1}, \tilde{\lambda}_{2}\right) \sum_{n=2}^{\infty}\left(1-O\left(\frac{1}{\ln ^{\widetilde{\lambda}_{1}} V_{n}}\right)\right) \frac{v_{n+1}}{V_{n} \ln ^{\varepsilon+1} V_{n}} \\
& =B\left(\tilde{\lambda}_{1}, \tilde{\lambda}_{2}\right)\left[\sum_{n=2}^{\infty} \frac{v_{n+1}}{V_{n} \ln ^{\varepsilon+1} V_{n}}-\sum_{n=2}^{\infty} O\left(\frac{v_{n+1}}{V_{n}\left(\ln V_{n}\right)^{\left(\frac{\varepsilon}{q}+\lambda_{1}\right)+1}}\right)\right] \\
& =\frac{1}{\varepsilon} B\left(\tilde{\lambda}_{1}, \tilde{\lambda}_{2}\right)\left[\frac{1}{\ln ^{\varepsilon}\left(1+v_{2}\right)}+\varepsilon(\widetilde{O}(1)-O(1))\right] \text {. }
\end{aligned}
$$

If there exists a positive constant $K \leq B\left(\lambda_{1}, \lambda_{2}\right)$ such that (38) is valid when replacing $B\left(\lambda_{1}, \lambda_{2}\right)$ by $K$, then, in particular, we have $\varepsilon \widetilde{I}<\varepsilon K\|\widetilde{a}\|_{p, \Omega_{\lambda}}\|\tilde{b}\|_{q, \digamma_{\lambda}}$, namely

$$
\begin{aligned}
& B\left(\widetilde{\lambda}_{1}, \tilde{\lambda}_{2}\right)\left[\frac{1}{\ln ^{\varepsilon}\left(1+v_{2}\right)}+\varepsilon(\widetilde{O}(1)-O(1))\right] \\
& \quad<K\left[\frac{1}{\ln ^{\varepsilon}\left(1+\mu_{2}\right)}+\varepsilon O(1)\right]^{\frac{1}{p}}\left[\frac{1}{\ln ^{\varepsilon}\left(1+v_{2}\right)}+\varepsilon \widetilde{O}(1)\right]^{\frac{1}{q}} .
\end{aligned}
$$


It follows that $B\left(\lambda_{1}, \lambda_{2}\right) \leq K\left(\varepsilon \rightarrow 0^{+}\right)$. Hence, $K=B\left(\lambda_{1}, \lambda_{2}\right)$ is the best possible constant factor of (38).

Similarly to (33), we still can find that

$$
I \leq J_{1}\|b\|_{q, \digamma_{\lambda}} .
$$

Hence, we can prove that the constant factor $B\left(\lambda_{1}, \lambda_{2}\right)$ in (39) is the best possible. Otherwise, we would reach a contradiction by (41) that the constant factor in (38) is not the best possible.

Remark 1 (i) It is evident that (38) and (39) are strengthened versions of the following equivalent Mulholland-type inequalities:

$$
\begin{aligned}
& \sum_{n=2}^{\infty} \sum_{m=2}^{\infty} \frac{a_{m} b_{n}}{\ln ^{\lambda}\left(U_{m} V_{n}\right)}<B\left(\lambda_{1}, \lambda_{2}\right)\|a\|_{p, \Phi_{\lambda}}\|b\|_{q, \Psi_{\lambda}}, \\
& \left\{\sum_{n=2}^{\infty} \frac{v_{n+1}}{V_{n}} \ln ^{p \lambda_{2}-1} V_{n}\left[\sum_{m=2}^{\infty} \frac{a_{m}}{\ln ^{\lambda}\left(U_{m} V_{n}\right)}\right]^{p}\right\}^{\frac{1}{p}}<B\left(\lambda_{1}, \lambda_{2}\right)\|a\|_{p, \Phi_{\lambda}},
\end{aligned}
$$

where the constant factor $B\left(\lambda_{1}, \lambda_{2}\right)$ is still the best possible.

(ii) For $\lambda=1, \lambda_{1}=\frac{1}{q}, \lambda_{2}=\frac{1}{p}$,

$$
\begin{aligned}
& \theta_{1}=\vartheta_{1}:=\frac{p \sin (\pi / p) \ln ^{1 / p}\left(1+v_{2} / 2\right)}{\pi\left[1+\frac{\ln \left(1+v_{2} / 2\right)}{\ln \left(1+\mu_{2} / 2\right)}\right]}, \\
& \theta_{2}=\vartheta_{2}:=\frac{q \sin (\pi / p) \ln ^{1 / q}\left(1+\mu_{2} / 2\right)}{\pi\left[1+\frac{\ln \left(1+\mu_{2} / 2\right)}{\ln \left(1+v_{2} / 2\right)}\right]},
\end{aligned}
$$

(38) reduces to the strengthened version of (9) as follows:

$$
\begin{aligned}
\sum_{m=2}^{\infty} \sum_{n=2}^{\infty} \frac{a_{m} b_{n}}{\ln U_{m} V_{n}}< & \frac{\pi}{\sin (\pi / p)}\left[\sum_{m=2}^{\infty}\left(1-\frac{\vartheta_{1}}{\ln ^{1 / p} U_{m}}\right)\left(\frac{U_{m}}{\mu_{m+1}}\right)^{p-1} a_{m}^{p}\right]^{\frac{1}{p}} \\
& \times\left[\sum_{n=2}^{\infty}\left(1-\frac{\vartheta_{2}}{\ln ^{1 / q} V_{n}}\right)\left(\frac{V_{n}}{v_{n+1}}\right)^{q-1} b_{n}^{q}\right]^{\frac{1}{q}} .
\end{aligned}
$$

For $\mu_{i}=v_{j}=1(i, j \in \mathbf{N}),(44)$ reduces to the following strengthened Mulholland's inequality:

$$
\begin{aligned}
\sum_{m=2}^{\infty} \sum_{n=2}^{\infty} \frac{a_{m} b_{n}}{\ln m n}< & \left\{\sum_{m=2}^{\infty}\left[\frac{\pi}{\sin (\pi / p)}-\frac{\ln \sqrt{3 / 2}}{\ln ^{1 / p} m}\right] \frac{a_{m}^{p}}{m^{1-p}}\right\}^{\frac{1}{p}} \\
& \times\left\{\sum_{n=2}^{\infty}\left[\frac{\pi}{\sin (\pi / p)}-\frac{\ln \sqrt{3 / 2}}{\ln ^{1 / q} m}\right] \frac{b_{n}^{q}}{n^{1-q}}\right\}^{\frac{1}{q}},
\end{aligned}
$$

where $\ln \sqrt{3 / 2}=0.20275^{+}$. 
For $p>1, \Psi_{\lambda}^{1-p}(n)=\frac{v_{n+1}}{V_{n}}\left(\ln V_{n}\right)^{p \lambda_{2}-1}$, we define the following normed spaces:

$$
\begin{aligned}
& l_{p, \Phi_{\lambda}}:=\left\{a=\left\{a_{m}\right\}_{m=2}^{\infty} ;\|a\|_{p, \Phi_{\lambda}}<\infty\right\}, \\
& l_{q, \Psi_{\lambda}}:=\left\{b=\left\{b_{n}\right\}_{n=2}^{\infty} ;\|b\|_{q, \Psi_{\lambda}}<\infty\right\}, \\
& l_{p, \Psi_{\lambda}^{1-p}}:=\left\{c=\left\{c_{n}\right\}_{n=2}^{\infty} ;\|c\|_{p, \Psi_{\lambda}^{1-p}}<\infty\right\} .
\end{aligned}
$$

Assuming that $a=\left\{a_{m}\right\}_{m=2}^{\infty} \in l_{p, \Phi_{\lambda}}$, setting

$$
c=\left\{c_{n}\right\}_{n=2}^{\infty}, c_{n}:=\sum_{m=2}^{\infty} \frac{a_{m}}{\ln ^{\lambda}\left(U_{m} V_{n}\right)}, \quad n \in \mathbf{N},
$$

we can rewrite (43) as follows:

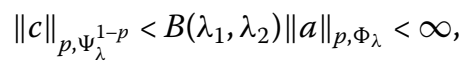

namely $c \in l_{p, \Psi_{\lambda}^{1-p}}$.

Definition 2 Define a Mulholland-type operator $T: l_{p, \Phi_{\lambda}} \rightarrow l_{p, \Psi_{\lambda}^{1-p}}$ as follows: For any $a=\left\{a_{m}\right\}_{m=2}^{\infty} \in l_{p, \Phi_{\lambda}}$, there exists a unique representation $T a=c \in l_{p, \Psi_{\lambda}^{1-p}}^{\lambda}$. Define the formal inner product of $T a$ and $b=\left\{b_{n}\right\}_{n=2}^{\infty} \in l_{q, \Psi_{\lambda}}$ as follows:

$$
(T a, b):=\sum_{n=2}^{\infty}\left[\sum_{m=2}^{\infty} \frac{a_{m}}{\ln ^{\lambda}\left(U_{m} V_{n}\right)}\right] b_{n} .
$$

Then we can rewrite (42) and (43) as follows:

$$
\begin{aligned}
& (T a, b)<B\left(\lambda_{1}, \lambda_{2}\right)\|a\|_{p, \Phi_{\lambda}}\|b\|_{q, \Psi_{\lambda}}, \\
& \|T a\|_{p, \Psi_{\lambda}^{1-p}}<B\left(\lambda_{1}, \lambda_{2}\right)\|a\|_{p, \Phi_{\lambda}} .
\end{aligned}
$$

Define the norm of operator $T$ as follows:

$$
\|T\|:=\sup _{a(\neq \theta) \in l_{p, \Phi_{\lambda}}} \frac{\|T a\|_{p, \Psi_{\lambda}^{1-p}}}{\|a\|_{p, \Phi_{\lambda}}} .
$$

Then by (43) we find $\|T\| \leq B\left(\lambda_{1}, \lambda_{2}\right)$. Since the constant factor in (48) is the best possible, we have

$$
\|T\|=B\left(\lambda_{1}, \lambda_{2}\right) .
$$

\section{Some strengthened versions of the reverses}

In the following, we also set

$$
\begin{aligned}
& \widetilde{\Omega}_{\lambda}(m):=\left(1-\theta\left(\lambda_{2}, m\right)\right) \frac{\left(\ln U_{m}\right)^{p\left(1-\lambda_{1}\right)-1}}{U_{m}^{1-p} \mu_{m+1}^{p-1}}, \\
& \widetilde{\digamma}_{\lambda}(n):=\left(1-\vartheta\left(\lambda_{1}, n\right)\right) \frac{\left(\ln V_{n}\right)^{q\left(1-\lambda_{2}\right)-1}}{V_{n}^{1-q} v_{n+1}^{q-1}} \quad(m, n \in \mathbf{N} \backslash\{1\}) .
\end{aligned}
$$


For $0<p<1$ or $p<0$, we still use the formal symbols $\|a\|_{p, \Phi_{\lambda}},\|b\|_{q, \Psi_{\lambda}},\|a\|_{p, \Omega_{\lambda}},\|b\|_{q, \digamma_{\lambda}}$, $\|a\|_{p, \widetilde{\Omega}_{\lambda}}$ and $\|b\|_{q, \widetilde{\digamma}_{\lambda}}$.

Theorem 3 If $0<p<1,\left\{\mu_{m}\right\}_{m=1}^{\infty}$ and $\left\{v_{n}\right\}_{n=1}^{\infty}$ are decreasing, $U_{\infty}=V_{\infty}=\infty,\|a\|_{p, \Phi_{\lambda}} \in \mathbf{R}_{+}$ and $\|b\|_{q, \Psi_{\lambda}} \in \mathbf{R}_{+}$, then we have the following equivalent inequalities with the best possible constant factor $B\left(\lambda_{1}, \lambda_{2}\right)$ :

$$
\begin{aligned}
& \sum_{n=2}^{\infty} \sum_{m=2}^{\infty} \frac{a_{m} b_{n}}{\ln ^{\lambda}\left(U_{m} V_{n}\right)}>B\left(\lambda_{1}, \lambda_{2}\right)\|a\|_{p, \widetilde{\Omega}_{\lambda}}\|b\|_{q, \digamma_{\lambda},} \\
& \left\{\sum_{n=2}^{\infty} \frac{v_{n+1} \ln ^{p \lambda_{2}-1} V_{n}}{\left(1-\frac{\theta_{2}}{\ln ^{\lambda} V_{n}}\right)^{p-1} V_{n}}\left[\sum_{m=2}^{\infty} \frac{a_{m}}{\ln ^{\lambda}\left(U_{m} V_{n}\right)}\right]^{p}\right\}^{\frac{1}{p}}>B\left(\lambda_{1}, \lambda_{2}\right)\|a\|_{p, \widetilde{\Omega}_{\lambda}} .
\end{aligned}
$$

Proof Using (22) and (17) in the reverses of (29) and (30), since

$$
\begin{aligned}
& \left(\omega\left(\lambda_{2}, m\right)\right)^{\frac{1}{p}}>\left(B\left(\lambda_{1}, \lambda_{2}\right)\right)^{\frac{1}{p}}\left(1-\theta\left(\lambda_{2}, m\right)\right)^{\frac{1}{p}} \quad(0<p<1), \\
& \left(\varpi\left(\lambda_{1}, n\right)\right)^{\frac{1}{q}}>\left(B\left(\lambda_{1}, \lambda_{2}\right)\right)^{\frac{1}{q}}\left(1-\frac{\theta_{2}}{\ln ^{\lambda_{1}} V_{n}}\right)^{\frac{1}{q}} \quad(q<0)
\end{aligned}
$$

and

$$
\frac{1}{\left(B\left(\lambda_{1}, \lambda_{2}\right)\right)^{p-1}\left(1-\frac{\theta_{2}}{V_{n}^{\lambda_{1}}}\right)^{p-1}}>\frac{1}{\left(\varpi\left(\lambda_{1}, n\right)\right)^{p-1}} \quad(0<p<1),
$$

we obtain equivalent inequalities (51) and (52).

For $\varepsilon \in\left(0, p \lambda_{1}\right)$, we set $\tilde{\lambda}_{1}, \tilde{\lambda}_{2}, \widetilde{a}_{m}$ and $\widetilde{b}_{n}$ as (40). Then, by (26), (27) and (17), we find

$$
\begin{aligned}
\|a\|_{p, \widetilde{\Omega}_{\lambda}}\|b\|_{q, \digamma_{\lambda}} & \geq\|a\|_{p, \widetilde{\Omega}_{\lambda}}\|b\|_{q, \Psi_{\lambda}} \\
= & {\left[\sum_{m=2}^{\infty}\left(1-\theta\left(\lambda_{2}, m\right)\right) \frac{\mu_{m+1}}{U_{m} \ln ^{1+\varepsilon} U_{m}}\right]^{\frac{1}{p}}\left(\sum_{n=2}^{\infty} \frac{v_{n+1}}{V_{n} \ln ^{1+\varepsilon} V_{n}}\right)^{\frac{1}{q}} } \\
= & \left(\sum_{m=2}^{\infty} \frac{\mu_{m+1}}{U_{m} \ln ^{1+\varepsilon} U_{m}}-\sum_{m=2}^{\infty} O\left(\frac{\mu_{m+1}}{U_{m} \ln ^{1+\lambda_{2}+\varepsilon} U_{m}}\right)\right)^{\frac{1}{p}} \\
& \times\left(\sum_{n=2}^{\infty} \frac{v_{n+1}}{V_{n} \ln ^{1+\varepsilon} V_{n}}\right)^{\frac{1}{q}} \\
= & \frac{1}{\varepsilon}\left[\frac{1}{\ln ^{\varepsilon}\left(1+\mu_{2}\right)}+\varepsilon\left(O(1)-O_{1}(1)\right)\right]^{\frac{1}{p}}\left[\frac{1}{\ln ^{\varepsilon}\left(1+v_{2}\right)}+\varepsilon \widetilde{O}(1)\right]^{\frac{1}{q}}, \\
\widetilde{I}:= & \sum_{n=2}^{\infty} \sum_{m=2}^{\infty} \frac{\tilde{a}_{m} \widetilde{b}_{n}}{\ln ^{\lambda}\left(U_{m} V_{n}\right)} \\
= & \sum_{n=2}^{\infty}\left[\sum_{m=2}^{\infty} \frac{1}{\ln ^{\lambda}\left(U_{m} V_{n}\right)} \frac{\mu_{m+1} \ln ^{\tilde{\lambda}_{2}} V_{n}}{U_{m} \ln ^{1-\widetilde{\lambda}_{1}} U_{m}}\right] \frac{v_{n+1}}{V_{n} \ln ^{\varepsilon+1} V_{n}}
\end{aligned}
$$




$$
\begin{aligned}
& =\sum_{n=2}^{\infty} \varpi\left(\widetilde{\lambda}_{1}, n\right) \frac{v_{n+1}}{V_{n} \ln ^{\varepsilon+1} V_{n}} \leq B\left(\widetilde{\lambda}_{1}, \tilde{\lambda}_{2}\right) \sum_{n=2}^{\infty} \frac{v_{n+1}}{V_{n} \ln ^{\varepsilon+1} V_{n}} \\
& =\frac{1}{\varepsilon} B\left(\widetilde{\lambda}_{1}, \widetilde{\lambda}_{2}\right)\left[\frac{1}{\ln ^{\varepsilon}\left(1+v_{2}\right)}+\varepsilon \widetilde{O}(1)\right] .
\end{aligned}
$$

If there exists a positive constant $K \geq B\left(\lambda_{1}, \lambda_{2}\right)$ such that (51) is valid when replacing $B\left(\lambda_{1}, \lambda_{2}\right)$ by $K$, then, in particular, we have $\varepsilon \widetilde{I}>\varepsilon K\|\widetilde{a}\|_{p, \widetilde{\Omega}_{\lambda}}\|\widetilde{b}\|_{q, \digamma_{\lambda}}$, namely

$$
\begin{aligned}
& B\left(\tilde{\lambda}_{1}, \tilde{\lambda}_{2}\right)\left[\frac{1}{\ln ^{\varepsilon}\left(1+v_{2}\right)}+\varepsilon \widetilde{O}(1)\right] \\
& \quad>K\left[\frac{1}{\ln ^{\varepsilon}\left(1+\mu_{2}\right)}+\varepsilon\left(O(1)-O_{1}(1)\right)\right]^{\frac{1}{p}}\left[\frac{1}{\ln ^{\varepsilon}\left(1+v_{2}\right)}+\varepsilon \widetilde{O}(1)\right]^{\frac{1}{q}} .
\end{aligned}
$$

It follows that $B\left(\lambda_{1}, \lambda_{2}\right) \geq K\left(\varepsilon \rightarrow 0^{+}\right)$. Hence, $K=B\left(\lambda_{1}, \lambda_{2}\right)$ is the best possible constant factor of (51).

The constant factor $B\left(\lambda_{1}, \lambda_{2}\right)$ in (52) is still the best possible. Otherwise, we would reach a contradiction by the reverse of (41) that the constant factor in (51) is not the best possible.

Remark 2 It is evident that (51) and (52) are strengthened versions of the following equivalent inequalities:

$$
\begin{aligned}
& \sum_{n=2}^{\infty} \sum_{m=2}^{\infty} \frac{a_{m} b_{n}}{\ln ^{\lambda}\left(U_{m} V_{n}\right)}>B\left(\lambda_{1}, \lambda_{2}\right)\|a\|_{p, \widetilde{\Omega}_{\lambda}}\|b\|_{q, \Psi_{\lambda}}, \\
& \left\{\sum_{n=2}^{\infty} \frac{v_{n+1}}{V_{n}} \ln ^{p \lambda_{2}-1} V_{n}\left[\sum_{m=2}^{\infty} \frac{a_{m}}{\ln ^{\lambda}\left(U_{m} V_{n}\right)}\right]^{p}\right\}^{\frac{1}{p}}>B\left(\lambda_{1}, \lambda_{2}\right)\|a\|_{p, \widetilde{\Omega}_{\lambda}},
\end{aligned}
$$

where the constant factor $B\left(\lambda_{1}, \lambda_{2}\right)$ is still the best possible.

Theorem 4 If $p<0,\left\{\mu_{m}\right\}_{m=1}^{\infty}$ and $\left\{v_{n}\right\}_{n=1}^{\infty}$ are decreasing, $U_{\infty}=V_{\infty}=\infty,\|a\|_{p, \Phi_{\lambda}} \in \mathbf{R}_{+}$ and $\|b\|_{q, \Psi_{\lambda}} \in \mathbf{R}_{+}$, then we have the following equivalent inequalities with the best possible constant factor $B\left(\lambda_{1}, \lambda_{2}\right)$ :

$$
\begin{aligned}
& \sum_{n=1}^{\infty} \sum_{m=1}^{\infty} \frac{a_{m} b_{n}}{\ln ^{\lambda}\left(U_{m} V_{n}\right)}>B\left(\lambda_{1}, \lambda_{2}\right)\|a\|_{p, \Omega_{\lambda}}\|b\|_{q, \tilde{\digamma}_{\lambda},} \\
& J_{2}:=\left\{\sum_{n=1}^{\infty} \frac{v_{n+1} \ln \ln ^{p \lambda_{2}-1} V_{n}}{\left(1-\vartheta\left(\lambda_{1}, n\right)\right)^{p-1} V_{n}}\left[\sum_{m=1}^{\infty} \frac{a_{m}}{\ln ^{\lambda}\left(U_{m} V_{n}\right)}\right]^{p}\right\}^{\frac{1}{p}}>B\left(\lambda_{1}, \lambda_{2}\right)\|a\|_{p, \Omega_{\lambda}} .
\end{aligned}
$$

Proof Using (16) and (23) in the reverses of (29) and (30), since

$$
\begin{aligned}
& \left(\omega\left(\lambda_{2}, m\right)\right)^{\frac{1}{p}}>\left(B\left(\lambda_{1}, \lambda_{2}\right)\right)^{\frac{1}{p}}\left(1-\frac{\theta_{1}}{U_{m}^{\lambda_{2}}}\right)^{\frac{1}{p}} \quad(p<0), \\
& \left(\varpi\left(\lambda_{1}, n\right)\right)^{\frac{1}{q}}>\left(B\left(\lambda_{1}, \lambda_{2}\right)\right)^{\frac{1}{q}}\left(1-\vartheta\left(\lambda_{1}, n\right)\right)^{\frac{1}{q}} \quad(0<q<1)
\end{aligned}
$$


and

$$
\left[\frac{1}{\left(B\left(\lambda_{1}, \lambda_{2}\right)\right)^{p-1}\left(1-\vartheta\left(\lambda_{1}, n\right)\right)^{p-1}}\right]^{\frac{1}{p}}>\left[\frac{1}{\left(\varpi\left(\lambda_{1}, n\right)\right)^{p-1}}\right]^{\frac{1}{p}} \quad(p<0),
$$

we obtain equivalent inequalities (55) and (56).

For $\varepsilon \in\left(0, q \lambda_{2}\right)$, we set $\tilde{\lambda}_{1}=\lambda_{1}+\frac{\varepsilon}{q}(>0), \tilde{\lambda}_{2}=\lambda_{2}-\frac{\varepsilon}{q}(\in(0,1))$, and

$$
\begin{aligned}
& \widetilde{a}_{m}:=\frac{\mu_{m+1}}{U_{m}} \ln ^{\tilde{\lambda}_{1}-\varepsilon-1} U_{m}=\frac{\mu_{m+1}}{U_{m}} \ln ^{\lambda_{1}-\frac{\varepsilon}{p}-1} U_{m}, \\
& \widetilde{b}_{n}=\frac{v_{n+1}}{V_{n}} \ln ^{\tilde{\lambda}_{2}-1} V_{n}=\frac{v_{n+1}}{V_{n}} \ln ^{\lambda_{2}-\frac{\varepsilon}{q}-1} V_{n} .
\end{aligned}
$$

Then, by (26), (27) and (16), we have

$$
\begin{aligned}
& \|\widetilde{a}\|_{p, \Omega_{\lambda}}\|\widetilde{b}\|_{q, \widetilde{\digamma}_{\lambda}} \\
& \geq\|\tilde{a}\|_{p, \Phi_{\lambda}}\|\widetilde{b}\|_{q, \tilde{\digamma}_{\lambda}} \\
& =\left(\sum_{m=2}^{\infty} \frac{\mu_{m+1}}{U_{m} \ln ^{\varepsilon+1} U_{m}}\right)^{\frac{1}{p}}\left[\sum_{n=2}^{\infty}\left(1-\vartheta\left(\lambda_{1}, n\right)\right) \frac{v_{n+1}}{V_{n} \ln ^{\varepsilon+1} V_{n}}\right]^{\frac{1}{q}} \\
& =\left(\sum_{m=2}^{\infty} \frac{\mu_{m+1}}{U_{m} \ln ^{\varepsilon+1} U_{m}}\right)^{\frac{1}{p}}\left[\sum_{n=2}^{\infty} \frac{v_{n+1}}{V_{n} \ln ^{\varepsilon+1} V_{n}}-\sum_{n=2}^{\infty} O\left(\frac{v_{n+1}}{V_{n} \ln ^{1+\left(\lambda_{1}+\varepsilon\right)} V_{n}}\right)\right]^{\frac{1}{q}} \\
& =\frac{1}{\varepsilon}\left[\frac{1}{\ln ^{\varepsilon}\left(1+\mu_{2}\right)}+\varepsilon O(1)\right]^{\frac{1}{p}}\left[\frac{1}{\ln ^{\varepsilon}\left(1+v_{2}\right)}+\varepsilon\left(\widetilde{O}(1)-O_{1}(1)\right)\right]^{\frac{1}{q}}, \\
& \widetilde{I}=\sum_{m=2}^{\infty} \sum_{n=2}^{\infty} \frac{\widetilde{a}_{m} \widetilde{b}_{n}}{\ln ^{\lambda}\left(U_{m} V_{n}\right)} \\
& =\sum_{m=2}^{\infty}\left[\sum_{n=2}^{\infty} \frac{\ln ^{\tilde{\lambda}_{1}} U_{m}}{\ln ^{\lambda}\left(U_{m} V_{n}\right)} \frac{v_{n+1}}{V_{n}} \ln ^{\tilde{\lambda}_{2}-1} V_{n}\right] \frac{\mu_{m+1}}{U_{m} \ln ^{\varepsilon+1} U_{m}} \\
& =\sum_{m=2}^{\infty} \omega\left(\tilde{\lambda}_{2}, m\right) \frac{\mu_{m+1}}{U_{m} \ln ^{\varepsilon+1} U_{m}} \leq B\left(\tilde{\lambda}_{1}, \tilde{\lambda}_{2}\right) \sum_{n=2}^{\infty} \frac{\mu_{m+1}}{U_{m} \ln ^{\varepsilon+1} U_{m}} \\
& =\frac{1}{\varepsilon} B\left(\tilde{\lambda}_{1}, \tilde{\lambda}_{2}\right)\left[\frac{1}{\ln ^{\varepsilon}\left(1+\mu_{2}\right)}+\varepsilon O(1)\right] \text {. }
\end{aligned}
$$

If there exists a positive constant $K \geq B\left(\lambda_{1}, \lambda_{2}\right)$ such that (55) is valid when replacing $B\left(\lambda_{1}, \lambda_{2}\right)$ by $K$, then, in particular, we have $\varepsilon \widetilde{I}>\varepsilon K\|\widetilde{a}\|_{p, \Omega_{\lambda}}\|\widetilde{b}\|_{q, \tilde{\digamma}_{\lambda}}$, namely

$$
\begin{aligned}
& B\left(\tilde{\lambda}_{1}, \tilde{\lambda}_{2}\right)\left[\frac{1}{\ln ^{\varepsilon}\left(1+\mu_{2}\right)}+\varepsilon O(1)\right] \\
& \quad>K\left[\frac{1}{\ln ^{\varepsilon}\left(1+\mu_{2}\right)}+\varepsilon O(1)\right]^{\frac{1}{p}}\left[\frac{1}{\ln ^{\varepsilon}\left(1+v_{2}\right)}+\varepsilon\left(\widetilde{O}(1)-O_{1}(1)\right)\right]^{\frac{1}{q}} .
\end{aligned}
$$

It follows that $B\left(\lambda_{1}, \lambda_{2}\right) \geq K\left(\varepsilon \rightarrow 0^{+}\right)$. Hence, $K=B\left(\lambda_{1}, \lambda_{2}\right)$ is the best possible constant factor of (55). 
Similarly to the reverse of (33), we still find that

$$
I \geq J_{2}\|b\|_{q, \widetilde{\digamma}_{\lambda}} .
$$

Hence the constant factor $B\left(\lambda_{1}, \lambda_{2}\right)$ in (56) is still the best possible. Otherwise, we would reach a contradiction by (57) that the constant factor in (55) is not the best possible.

Remark 3 It is evident that (55) and (56) are strengthened versions of the following equivalent inequalities:

$$
\begin{aligned}
& \sum_{n=2}^{\infty} \sum_{m=2}^{\infty} \frac{a_{m} b_{n}}{\ln ^{\lambda}\left(U_{m} V_{n}\right)}>B\left(\lambda_{1}, \lambda_{2}\right)\|a\|_{p, \Phi_{\lambda}}\|b\|_{q, \widetilde{\digamma}_{\lambda},} \\
& \left\{\sum_{n=2}^{\infty} \frac{v_{n+1} \ln ^{p \lambda_{2}-1} V_{n}}{\left(1-\vartheta\left(\lambda_{1}, n\right)\right)^{p-1} V_{n}}\left[\sum_{m=2}^{\infty} \frac{a_{m}}{\ln ^{\lambda}\left(U_{m} V_{n}\right)}\right]^{p}\right\}^{\frac{1}{p}}>B\left(\lambda_{1}, \lambda_{2}\right)\|a\|_{p, \Phi_{\lambda}},
\end{aligned}
$$

where the constant factor $B\left(\lambda_{1}, \lambda_{2}\right)$ is still the best possible.

\section{Competing interests}

The authors declare that they have no competing interests.

\section{Authors' contributions}

BY carried out the mathematical studies, participated in the sequence alignment and drafted the manuscript. AW and QH participated in the design of the study and performed the numerical analysis. All authors read and approved the final manuscript.

\section{Acknowledgements}

The authors wish to express their thanks to the referees for their careful reading of the manuscript and for their valuable suggestions. This work is supported by the National Natural Science Foundation (No. 61370186), and 2013 Knowledge Construction Special Foundation Item of Guangdong Institution of Higher Learning College and University (No. 2013KJCX0140)

Received: 5 August 2015 Accepted: 30 September 2015 Published online: 12 October 2015

\section{References}

1. Hardy, GH, Littlewood, JE, Pólya, G: Inequalities. Cambridge University Press, Cambridge (1934)

2. Mulholland, HP: Some theorems on Dirichlet series with positive coefficients and related integrals. Proc. Lond. Math. Soc. 29(2), 281-292 (1929)

3. Mitrinović, DS, Pečarić, JE, Fink, AM: Inequalities Involving Functions and Their Integrals and Derivatives. Kluwer Academic, Boston (1991)

4. Yang, BC: Hilbert-Type Integral Inequalities. Bentham Science Publishers, Sharjah (2009)

5. Yang, BC: Discrete Hilbert-Type Inequalities. Bentham Science Publishers, Sharjah (2011)

6. Yang, BC: On Hilbert's integral inequality. J. Math. Anal. Appl. 220, 778-785 (1998)

7. Yang, BC: An extension of Mulholland's inequality. Jordan J. Math. Stat. 3(3), 151-157 (2010)

8. Yang, BC: The Norm of Operator and Hilbert-Type Inequalities. Science Press, Beijing (2009)

9. Gao, MZ, Yang, BC: On the extended Hilbert's inequality. Proc. Am. Math. Soc. 126(3), 751-759 (1998)

10. Yang, BC: An extension of a Hardy-Hilbert-type inequality. J. Guangdong Univ. Educ. 35(3), 1-7 (2015)

11. Wang, DX, Guo, DR: Introduction to Spectral Functions. Science Press, Beijing (1979)

12. Kuang, JC: Applied Inequalities. Shangdong Science Technic Press, Jinan (2004) 